NEUROGENETICS

\section{Schizophrenia: missing heritability found?}

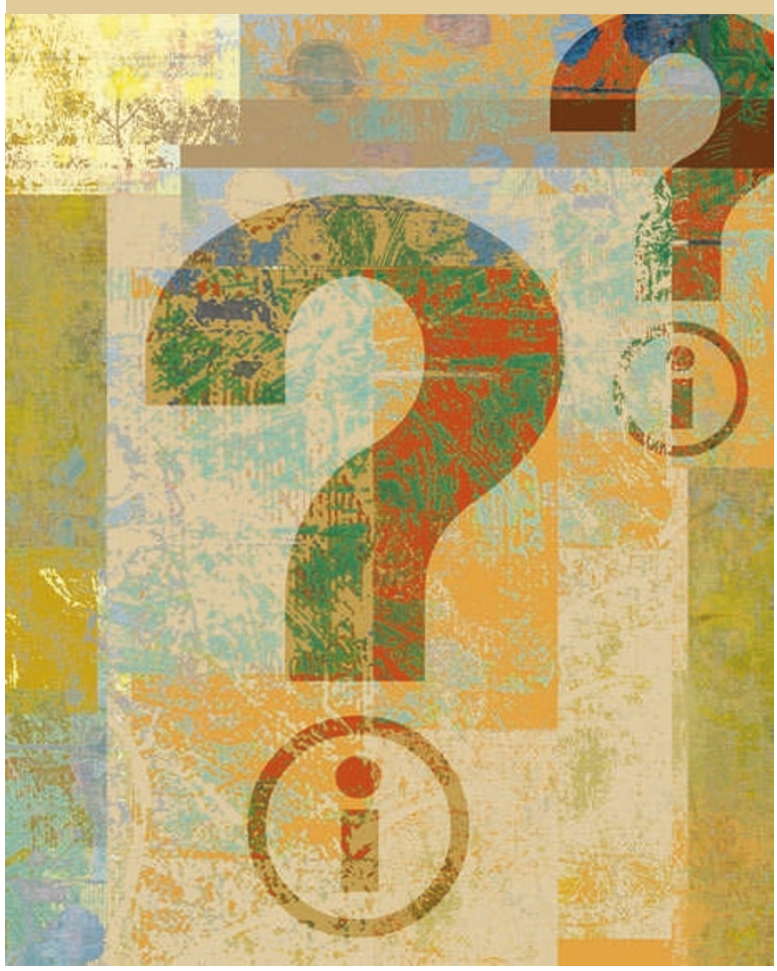

Although genome-wide association (GWA) studies for complex diseases or traits have now been with us for a number of years, mental disorders have proved particularly resistant to this approach. Conventional wisdom has been that problems with exact phenotyping will always make GWA studies difficult for mental disorders. We know that there is a heritable component to these disorders, but genetic studies have largely been unable to agree on the exact loci for association and follow-up. Three new studies of schizophrenia illustrate some of the progress made by using GWA studies to investigate neuropsychiatric conditions, but they also highlight the utility of alternative approaches.

Thanks to the sharing of data between three large consortia, a metaanalysis was carried out on GWA study results from over 8,000 cases of schizophrenia and 19,000 controls of European descent. All three resulting papers report that only through the very large meta-analysis were the researchers able to see associations between schizophrenia and single nucleotide polymorphisms (SNPs) in or close to the major histocompatibility complex (MHC) on chromosome 6. Both Stefansson et al. and Shi et al. suggest a number of intriguing explanations for their findings. In particular, Stefansson et al. speculate that schizophrenia is a response to infection or that disease risk is related to month of birth. However, a definitive biological mechanism linking the disease to MHC function remains to be elucidated. It is also noteworthy that, using GWA studies, other diseases have shown 'hits' in the MHC, including neurological conditions (Alzheimer's disease) and autoimmune disorders (Crohn's disease, both types of diabetes and multiple sclerosis).

At the same time, the International Schizophrenia Consortium used a second approach: here, a test for polygenic inheritance was used instead of a conventional GWA study. This involved searching for genetic association under a model of several thousand alleles of very small effect that combine to produce a testable, quantitative 'score'. A large number of alleles, with weak individual association to schizophrenia, pointed to a highly polygenic model; these alleles were not shared with any non-psychiatric disorder tested but they did overlap with those associated with bipolar disorder. Furthermore, the common variants tagged by these markers may, as a whole, contribute at least one-third of the heritability of schizophrenia.

A year ago, studies reported that copy number variants (CNVs) at several chromosomal locations were associated with schizophrenia. Although the mechanisms by which SNPs and CNVs contribute to genetic association may be different, the limited - if any - overlap between the new SNP results and the CNV associations contributes to the ongoing debate in genetics about the 'missing heritability': despite large GWA studies we are still far from explaining the estimated heritability of most complex diseases. Future studies examining different genetic architectures, including polygenic models, may bring the answer.

Chris Gunter, HudsonAlpha Institute for Biotechnology

\section{ORIGINAL RESEARCH PAPERS}

The International Schizophrenia Consortium. Common polygenic variation contributes to risk of schizophrenia and bipolar disorder. Nature 1 Jul 2009 (doi:10.1038/nature08185)| Stefansson, H. et al. Common variants conferring risk of schizophrenia. Nature 1 Jul 2009 (doi:10.1038/ nature08186)|Shi, J. et al. Common variants on chromosome $6 \mathrm{p} 22.1$ are associated with schizophrenia. Nature 1 Jul 2009 (doi:10.1038/ nature08192)

FURTHER READING Maher, B. Personal genomes: the case of the missing heritability. Nature 456, 18-21 (2008) 